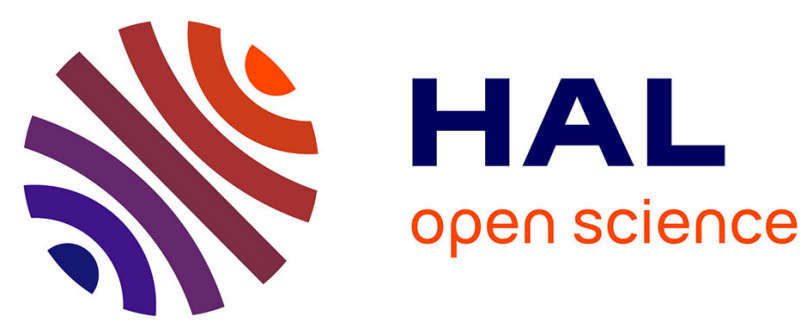

\title{
Urinary collecting system invasion is an independent prognostic factor of organ confined renal cell carcinoma.
}

Grégory Verhoest, Raffi Avakian, Karim Bensalah, Rodolphe Thuret, Vincenzo Ficarra, Walter Artibani, Jacques Tostain, François Guille, Lucas Cindolo, Alexandre de La Taille, et al.

\section{To cite this version:}

Grégory Verhoest, Raffi Avakian, Karim Bensalah, Rodolphe Thuret, Vincenzo Ficarra, et al.. Urinary collecting system invasion is an independent prognostic factor of organ confined renal cell carcinoma.. Journal of Urology, 2009, 182 (3), pp.854-9. 10.1016/j.juro.2009.05.017 . inserm-00410865

\section{HAL Id: inserm-00410865 https://www.hal.inserm.fr/inserm-00410865}

Submitted on 3 Sep 2009

HAL is a multi-disciplinary open access archive for the deposit and dissemination of scientific research documents, whether they are published or not. The documents may come from teaching and research institutions in France or abroad, or from public or private research centers.
L'archive ouverte pluridisciplinaire HAL, est destinée au dépôt et à la diffusion de documents scientifiques de niveau recherche, publiés ou non, émanant des établissements d'enseignement et de recherche français ou étrangers, des laboratoires publics ou privés. 


\title{
Urinary Collecting System Invasion is an Independent Prognostic
}

\section{Factor in Organ Confined Renal Cell Carcinomas.}

\author{
Grégory VERHOEST ${ }^{1}$, Raffi AVAKIAN ${ }^{1}$, Karim BENSALAH ${ }^{1}$, Rodolphe THURET $^{2}$, \\ Vincenzo FICARRA ${ }^{3}$, Walter ARTIBANI ${ }^{3}$, Jacques TOSTAIN ${ }^{4}$, François GUILLE ${ }^{1}$, Lucas \\ CINDOLO $^{5}$, Alexandre DE LA TAILLE ${ }^{6}$, Claude-Clément ABBOU ${ }^{6}$, Laurent SALOMON $^{6}$, \\ Nathalie RIOUX-LECLERCQ ${ }^{1}$, Jean-Jacques PATARD ${ }^{1}$ \\ ${ }^{1}$ Rennes University Hospital, France \\ ${ }^{2}$ Montpellier University Hospital, France \\ ${ }^{3}$ Padova University Hospital, Italy \\ ${ }^{4}$ Saint Etienne University, France \\ ${ }^{5}$ G. Rummo Hospital, Benevento, Italy. \\ ${ }^{6}$ Henri Mondor University Hospital,, Créteil, France
}

Corresponding author:

Grégory VERHOEST, MD

Department of Urology

Rennes University Hospital

35033 RENNES CEDEX, FRANCE

gregory.verhoest@wanadoo.fr

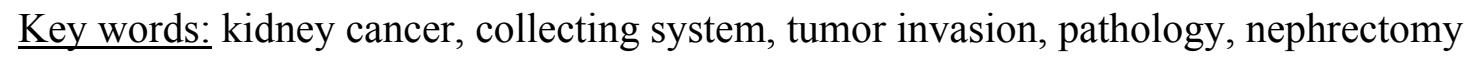




\section{Abstract:}

Objective: To evaluate urinary collecting system invasion (UCSI) as a prognostic parameter in renal cell carcinoma.

Material and methods: 1124 patients who underwent a nephrectomy for a renal tumor at 5 European centres were included in this retrospective study. The following variables were analysed: UCSI, age, sex, TNM stage, Fuhrman grade, histologic subtype, ECOG performance status (PS) and cancer specific survival.

Results: There were 771 males (68.6\%) and 353 females (31.4\%). Median age was 61 years [14-88]. Median tumor size was $6 \mathrm{~cm}$ [1-24]. Tumors were organ confined and Furhman grade was recorded as I-II in $67.1 \%$ and $62.3 \%$ of the cases, respectively. Symptoms were present at diagnosis and ECOG-PS was $\geq 1$ in $50.3 \%$ and $16.1 \%$ of the cases, respectively. At the end of follow-up 246 patients $(21.9 \%)$ died from cancer. In 132 cases (11.7\%), UCSI was noted. UCSI was associated with symptoms, TNM stage, Fuhrman grade, tumor size ( $<<0.001$ ), ECOG-PS (p: 0.003), but not with histologic subtype (p: 0.7). In univariate analysis: TNM stage, Fuhrman grade, symptoms, ECOG-PS, tumor size, and UCSI (p: 0.0001) were significant predictors for cancer specific survival. UCSI was found to be an independent prognostic parameter only in the setting of pT1-T2 tumors. When urinary collecting system was invaded, 5 and 10 years probabilities of survival were $43 \%$ and $41 \%$ respectively.

Conclusion: UCSI appears as an independent prognostic parameter in organ confined RCCs. Our data support the need to integrate this parameter in further TNM revisions. 


\section{Introduction:}

Renal cell carcinoma (RCC) represents $2-3 \%$ of all solid neoplasms and is the third most common genito-urinary tumor. Clear cell histological subtype accounts for $85 \%$ of the RCC cases ${ }^{1}$ while the annual incidence of renal tumors is increasing in by around $3 \%$ (1.5$5 \%$ ). This may be due in part to the widespread use of imaging techniques such as ultrasound (US) and computerized tomography $(\mathrm{CT})^{2}$ which allows the early detection of an increasing number of small renal masses.

The value of an accurate cancer staging system is crucial, not only for defining specific prognostic tools that will identify groups with greater risk of recurrence but also for providing cost-effective post-therapeutic surveillance protocols. TNM staging system remains the most useful determinant in disease classification reflecting the propensity of these tumors to extend locally $(\mathrm{T})$, involve regional lymph nodes $(\mathrm{N})$ and initiate metastatic growth $(\mathrm{M})^{3}$. Although invasion of Gerota's fascia, perirenal fat, adrenal gland, renal vein, inferior vena cava, and adjacent retroperitoneal structures have been extensively studied and discussed as prognostic variables, the prognostic value of the urinary collecting system invasion (UCSI) has not focused great interest in the recent RCC prognostication literature ${ }^{4}$. Invasion of the urinary collecting system, mentioned in the 1978 edition of the TNM classification, is no longer considered as a criterion for staging $\mathrm{RCC}^{5}$. However, some recent studies suggested that UCSI could be potentially interesting as a prognostic variable in low stage tumors ${ }^{6}$.

The aim of this large multicenter retrospective study was to assess the relationship between UCSI and usual prognostic variables in RCC and to evaluate to what extent UCSI should be considered as a major prognostic variable in RCC leading to further change of the TNM classification. 


\section{Material and Methods}

\section{Data retrieval}

This retrospective study included 1124 patients who underwent either radical nephrectomy or nephron sparing surgery for a renal tumor between 1997 and 2004. Data were extracted from RCC databases of 5 European academic centres: Rennes, Créteil, Saint-Etienne (France), Padua and Benevento (Italy). The study was approved by the IRB at each institution. Only tumors with malignant histology at final pathology were retained for analysis. Patients with metastatic disease were treated by radical nephrectomy followed by interferon, interleukin 2based immunotherapy according to the standards of care at that time. Post-operative follow-up was established according to protocols at each institution.

\section{$\underline{\text { Clinical and histological variables. }}$}

Involvement of the urinary collecting system (UCS) was defined as evidence of invasion of the calices, infundibulum, pelvis or ureter; excluding simple compression or displacement by the primary renal mass. Tumor stage and grade were determined according to the 2002 TNM classification ${ }^{5}$, and Fuhrman grading system respectively ${ }^{7}$. Histologic subtypes were stratified according to the World Health Organization classification ${ }^{8}$. All the following variables were recorded and analysed: age, gender, presence of symptoms at diagnosis, Eastern Cooperative Oncology Group (ECOG) Performance Status (PS), tumor size, TNM stage, Fuhrman grade, UCSI, histologic subtype, and survival data. At last follow up, patients were considered as living (with or without disease) or deceased (from renal cancer or from any other cause). 


\section{Statistical analysis:}

For statistical analysis, patients were classified as ECOG 0 versus 1 or higher. Qualitative and quantitative variables were compared by using Chi-square (Fischer exact test) and Student $t$ tests, respectively. All analyses were conducted with the statistical Package for the Social Sciences version 13.1 (SPSS Inc, Chicago, IL, USA), and $p$ value significance was fixed at 0.05. Univariate and multivariate analyses were performed by using the Kaplan-Meier and the Cox methods respectively. 


\section{$\underline{\text { Results }}$}

\section{$\underline{\text { Patients and tumor characteristics }}$}

This study included 1124 patients; out of them 771 were males (68.6\%) and 353 were females (31.4\%). Median age was 61 years [14-88]. Symptoms were present at diagnosis in $50.3 \%$ of the cases. Median tumor size was $6 \mathrm{~cm}$ [1-24]. Tumors were organ confined and recorded as Fuhrman grade 1 or 2 in $67.2 \%$ and $62.3 \%$ of the cases respectively. Surgical procedure was a radical nephrectomy in 896 cases $(79.7 \%)$. The most common histopathological diagnosis was clear cell carcinoma in 875 cases (84\%). Mean follow-up was 63 months. At the end of the follow-up, 246 patients (21.9\%) died from cancer. Patient and tumor characteristics are summarized in Table 1. One hundred and thirty two patients $(11.7 \%)$ had pathological evidence of UCSI.

\section{$\underline{\text { Relationship between UCSI and clinico-pathological features }}$}

UCSI was clearly associated with aggressive clinico-pathological features. For example, when symptoms were present at diagnosis, UCSI was observed in $17.6 \%$ of the cases vs only $4.6 \%$ in incidental tumors. Similarly, when ECOC PS was recorded as 1 or more, $17.4 \%$ of the tumors invaded UCS vs $9.5 \%$ in case of ECOG 0 . Locally advanced (T Stage $\geq 3$ ) and high grade tumors (Fuhrman grade $\geq 3$ ) were associated with more frequent UCSI $(26.2 \%$ and 23.1 $\%)$ compared to organ confined and low grade tumors ( $4.6 \%$ in both cases, $p<0.001)$. In case of nodal invasion or distant metastases, UCSI was also more frequently noted $(34.3 \%$ and $22.3 \%, p<0.001)$. However, histological subtype had no impact on UCSI. All results regarding relationship between UCSI and clinico-pathological features are summarized in table 2 . 


\section{$\underline{\text { Survival analysis }}$}

Evaluation of survival outcome of patients without UCSI revealed that cancer specific survival at 5 and 10 years was $80 \%$ and $74 \%$ respectively, while patients with UCSI had $43 \%$ and $41 \%$ cancer specific survival rates at 5 and 10 years respectively (Figure 1). Median survival time was not reached for patients without UCSI, whereas it was 60 months when the collecting system was invaded $(p=0.0001)$. Sub stratifying T stage with or without UCSI revealed a significant survival difference either for patients with pT1-T2 tumors $(p=0.0001)$, or for those with pT3-T4 tumors $(p=0.04)$ (Figure 2 ). When comparing pT2 tumors with UCSI to pT3 tumors there was no survival difference $(p=0.95)$. In univariate analysis, TNM stage, Fuhrman grade, symptoms at diagnosis, ECOG-PS, median tumor size and UCSI $(p<0.001)$ appeared as significant prognostic variables for cancer specific survival (Table 3).

In multivariate analysis, TNM stage, median tumor size $(p<0.001)$, ECOG-PS $(p=0.002)$ and Fuhrman grade $(p=0.02)$ were independent prognostic factors. Interestingly, UCSI was not retained as an independent prognostic factor for the entire population (Table 3). However, when searching separately for independent prognostic factors in pT1-T2 and pT3-T4 tumors, it appeared that UCSI was an independent prognostic variable in pT1-T2 tumors while it was not in pT3-T4 tumors (tables 4 and 5). When both ECOG performance status and symptoms at diagnosis were included within the organ confined Cox model, only ECOG was retained as an independent prognostic parameter. When symptoms at diagnosis were included within the model but not ECOG performance status; both UCSI $(p=0.02)$ and symptoms at presentation $(p=0.002)$ were retained as independent prognostic parameters. Consistently with the current 2002 TNM classification, perinephritic fat invasion $(p<0.001)$ and renal vein invasion $(p=$ 0.01) were retained as independent prognostic factors in the setting of pT3-T4 tumors. Median 
tumor size was also found to be an additional independent prognostic parameter in pT3-T4 tumors (Table 6). 


\section{$\underline{\text { Discussion }}$}

There is a consensus that TNM classification needs to be continuously revised according to the current state of the art in prognostication ${ }^{9}$. Large retrospective databases allow such a comprehensive analysis of anatomic and pathologic parameters that are the basis of the TNM classification. For example, the optimal tumor size cut-off for delineating T1 from T2 tumors has been extensively discussed ${ }^{10}$. Similarly, T3 sub classification of the 2002 TNM classification has been extensively challenged with discussion about prognostic value of perinephritic fat invasion versus sinus fat invasion ${ }^{11}$, adrenal gland invasion ${ }^{12}$, renal vein and the level of IVC invasion ${ }^{13}$. By contrast, prognostic value of UCSI has been poorly investigated so far.

To our knowledge, our series is the largest series published in the literature, with a cohort of 1124 patients coming from 5 European academic centers and including 132 tumors invading the collecting system (11.7\%). UCSI frequency that we reported is consistent with the range of the literature going from 7.5 to $14 \%{ }^{14-17}$. We thus demonstrated that tumors with UCSI presented with higher stages and grades and were more likely to invade lymph nodes or exhibit distant metastases. Consistently, these tumors which were larger in size were more likely to be symptomatic. Interestingly, although UCSI was not retained as an independent prognostic parameter overall, it was retained as an independent prognostic variable in $\mathrm{pT} 1-\mathrm{T} 2$ stage tumors. It suggests that pathological parameters such as renal vein invasion or perinephritic fat invasion are more important outcome predictors than UCSI in locally advanced tumors. Conversely, it suggests that UCSI, together with tumor size, ECOG performance status and symptoms could be an additional important prognostic variable in organ confined renal tumors. Interestingly we previously demonstrated that symptoms as well as tumor size were able to stratify T1-T2 tumors ${ }^{18}$. This additional data could provide some rationale for using tumor size, symptoms and UCSI for sub stratifying T1-T2 tumors similarly 
to that of fat, adrenal and renal vein invasion which are defining T3a, b and c stages in locally advanced tumors. We and others also demonstrated that tumors associated with symptoms at diagnosis were larger in size, of higher grades and exhibited poorer outcome ${ }^{19}$. Therefore, local symptoms such as haematuria could be viewed as surrogate indicators for UCSI. However, interestingly the present study highlighted the independent prognostic value of pathological invasion of UCS even after adjustment for tumor size, ECOG performance status or symptoms. It means that UCSI has an intrinsic biological significance in organ confined tumors, similarly to that of renal vein or capsular invasion in locally advanced disease. A tumor crossing an anatomic natural barrier such as collecting system should not be longer considered as an organ confined tumor. This is supported by the fact that we found no survival difference between pT2 tumors exhibiting UCSI and pT3 tumors. It should therefore be taken into account for further TNM classification.

Only 4 studies have focused on UCSI prognostic significance so far. In 2002, Uzzo et al analyzed the outcome of 61 patients with tumors invading UCS in $\mathrm{RCC}^{14}$. They concluded that these tumors although exhibiting higher stages and grades had not a significantly poorer outcome. It was noticed that even though UCSI in organ confined RCCs was uncommon, there was a trend for a worse prognosis when it occurred. However, the independent prognostic value of UCSI was not assessed through multivariate analyses in this study. By analyzing 124 tumors invading the UCS in 895 patients, Palapattu et al reported a high proportion of large (2/3 were classified as pT3) and symptomatic tumors within this population. UCSI was also significantly associated with lymph node invasion and presence of distant metastases at diagnosis ${ }^{15}$. Univariate analysis revealed a 1.96 time increased risk of death for patients with UCSI. Interestingly, the authors noticed a significantly worse prognosis in patients with T1 and T2 tumors with UCSI. Recently, the same group analyzed the outcome of 39 patients with UCSI in stage I and II RCCs ${ }^{17}$. No association was found 
between UCSI, T stage, Fuhrman grade, tumor size, ECOG-PS, capsular involvement and histological type. Univariate analysis confirmed a worse prognosis when UCS was invaded, resulting in a significantly poorer survival in this subgroup of patients. Furthermore consistently with our study, multivariate analysis revealed UCSI as an independent prognostic factor within organ confined tumors, with a 3.78 times increased risk of tumor recurrence. Finally, Terrone et al obtained comparable results except that adverse outcome associated with UCSI was only observed within the pT2 category ${ }^{16}$. The current series dealing with UCSI prognostic value are summarized in table 7.

Even though TNM staging remains the most widely recognized prognostic system it is important to further identify independent prognostic variables that are able either to improve the system itself or that can be integrated within more sophisticated prognostic tools. The common goal of identifying new prognostic variables is to improve the predictive accuracy of current prognostic systems that we dispose. Based on our results, we believe that UCSI could fit with such an objective for organ confined tumors. Based on the current TNM classification, organ confined renal tumors are only stratified based on tumor size. No reliable biological prognostic variable has been indentified so far in the setting of localized RCC. Therefore, information regarding UCSI could be either integrated within further TNM classification revision or being tested for incorporated within new nomograms specially designed for localized RCC. Finally, our study besides new insights in localized RCC prognostication also suggests that a closer follow up should be proposed for those patients with organ confined tumors and UCSI by contrast with what is usually proposed in such low stage RCCs ${ }^{20}$.

Our study is not exempt of limitations. The main drawback is that due to its retrospective character and to the lack of central pathological review, some critical information may have been missed. Notably, it could be argued that since UCSI has not been 
prospectively searched, only massive UCSI associated with large tumors has been reported. Then less obvious UCSI either microscopic or limited may have been missed even though it was present, therefore leading to a selection bias of more aggressive tumors. Additionally, because our study was based on retrospective pathological reports analysis, it could be hypothesized that this information was more likely to be noted in larger tumors that had been treated by radical nephrectomy rather than in small tumours treated by nephron sparing surgery. Our low NSS rate pleads for this hypothesis. Therefore by searching for CSI information based on the current pathological analysis practice we biased for larger tumors which are more likely to be symptomatic. Our 50\% symptomatic tumors rate which is a little bit higher than usually reported is also consistent with this hypothesis. Finally, it could also be argued that less attention is paid for CSI in small renal tumor specimens and that CSI is more difficult to assess following partial nephrectomy. It is probably true, however even though the size of tumor margins does not matter, careful examination of the quality of the tumor surrounding tissue is usually performed. Therefore, the presence or absence of urothelium tissue along with its invasion should be easily identified. Urologists should also mention whether or not collecting system has been entered and if there is any CSI suspicion. 


\section{Conclusion}

Tumors with UCSI are associated with most adverse clinical and pathological parameters in RCC. Based on the present study, we recommend that urinary collecting system invasion should be systematically identified and recorded in pathological reports. UCSI appears as a new important prognostic factor for organ confined tumors. This pathologic parameter should be considered for further TNM classification revisions and for its integration within new prognostic nomograms. Finally, a closer follow-up should be proposed to patients operated on for organ confined tumors and exhibiting UCSI. To what extend organ confined tumors with associated UCSI should be considered as locally advanced tumors and proposed for adjuvant trials needs to be further explored. 


\section{Bibliography:}

1. Pantuck, A. J., Zisman, A., Belldegrun, A.: The changing natural history of renal cell carcinoma. J Urol, 166: 1611, 2001

2. Patard, J. J., Tazi, H., Bensalah, K., Rodriguez, A., Vincendeau, S., RiouxLeclercq, N. et al.: The changing evolution of renal tumours: a single center experience over a two-decade period. Eur Urol, 45: 490, 2004

3. Ficarra, V., Schips, L., Guille, F., Li, G., De La Taille, A., Prayer Galetti, T. et al.: Multiinstitutional European validation of the 2002 TNM staging system in conventional and papillary localized renal cell carcinoma. Cancer, 104: 968, 2005

4. Ficarra, V., Guille, F., Schips, L., de la Taille, A., Prayer Galetti, T., Tostain, J. et al.: Proposal for revision of the TNM classification system for renal cell carcinoma. Cancer, 104: 2116, 2005

5. Sobin, L. H., Wittekind, C.: TNM. Classification of malignant tumors. Sixth Edition. UICC International Union Against Cancer, Ed Willey-Liss.: 193, 2003

6. Ficarra, V., Galfano, A., Mancini, M., Martignoni, G., Artibani, W.: TNM staging system for renal-cell carcinoma: current status and future perspectives. Lancet Oncol, 8: 554, 2007

7. Fuhrman, S. A., Lasky, L. C., Limas, C.: Prognostic significance of morphologic parameters in renal cell carcinoma. Am J Surg Pathol, 6: 655, 1982

8. Lopez-Beltran, A., Scarpelli, M., Montironi, R., Kirkali, Z.: 2004 WHO classification of the renal tumors of the adults. Eur Urol, 49: 798, 2006

9. Gospodarowicz, M. K., Miller, D., Groome, P. A., Greene, F. L., Logan, P. A., Sobin, L. H.: The process for continuous improvement of the TNM classification. Cancer, 100: 1, 2004

10. Karakiewicz, P. I., Lewinshtein, D. J., Chun, F. K., Briganti, A., Guille, F., Perrotte, P. et al.: Tumor Size Improves the Accuracy of TNM Predictions in Patients with Renal Cancer. Eur Urol, 50: 521, 2006

11. Thompson, R. H., Leibovich, B. C., Cheville, J. C., Webster, W. S., Lohse, C. M., Kwon, E. D. et al.: IS RENAL SINUS FAT INVASION THE SAME AS PERINEPHRIC FAT INVASION FOR pT3a RENAL CELL CARCINOMA? J Urol, 174: 1218,2005

12. Thompson, R. H., Leibovich, B. C., Cheville, J. C., Lohse, C. M., Frank, I., Kwon, E. D. et al.: Should direct ipsilateral adrenal invasion from renal cell carcinoma be classified as pT3a? J Urol, 173: 918, 2005

13. Ficarra, V., Novara, G., Iafrate, M., Cappellaro, L., Bratti, E., Zattoni, F. et al.: Proposal for reclassification of the TNM staging system in patients with locally 
advanced (pT3-4) renal cell carcinoma according to the cancer-related outcome. Eur Urol, 51: 722, 2007

14. Uzzo, R. G., Cherullo, E. E., Myles, J., Novick, A. C.: Renal cell carcinoma invading the urinary collecting system: implications for staging. J Urol, 167: 2392, 2002

15. Palapattu, G. S., Pantuck, A. J., Dorey, F., Said, J. W., Figlin, R. A., Belldegrun, A. S.: Collecting system invasion in renal cell carcinoma: impact on prognosis and future staging strategies. J Urol, 170: 768, 2003

16. Terrone, C., Cracco, C., Guercio, S., Bollito, E., Poggio, M., Scoffone, C. et al.: Prognostic value of the involvement of the urinary collecting system in renal cell carcinoma. Eur Urol, 46: 472, 2004

17. Klatte, T., Chung, J., Leppert, J. T., Lam, J. S., Pantuck, A. J., Figlin, R. A. et al.: Prognostic relevance of capsular involvement and collecting system invasion in stage I and II renal cell carcinoma. BJU Int, 99: 821, 2007

18. Patard, J. J., Dorey, F. J., Cindolo, L., Ficarra, V., De La Taille, A., Tostain, J. et al.: Symptoms as well as tumor size provide prognostic information on patients with localized renal tumors. J Urol, 172: 2167, 2004

19. Patard, J. J., Leray, E., Cindolo, L., Ficarra, V., Rodriguez, A., De La Taille, A. et al.: Multi-institutional validation of a symptom based classification for renal cell carcinoma. J Urol, 172: 858, 2004

20. Stephenson, A. J., Chetner, M. P., Rourke, K., Gleave, M. E., Signaevsky, M., Palmer, B. et al.: Guidelines for the surveillance of localized renal cell carcinoma based on the patterns of relapse after nephrectomy. J Urol, 172: 58, 2004 